\title{
Spatial character of polychlorinated biphenyls from soil and respirable particulate matter in Taiyuan, China
}

\author{
Shan $\mathrm{Fu}^{\mathrm{a}}$, Hang-Xin Cheng ${ }^{\mathrm{b}}$, Ying-Han Liu ${ }^{\mathrm{b}}$, Zhong-Zhi Yang ${ }^{\mathrm{a}}$, Xiao-Bai Xu ${ }^{\mathrm{a}, *}$ \\ a State Key Laboratory of Environmental Chemistry and Ecotoxicology, Research Center for Eco-Environmental Sciences, \\ Chinese Academy of Sciences, P.O. Box 2871, Beijing 100085, People's Republic of China \\ b Institute of Geophysical and Geochemical Exploration, Chinese Academy of Geological Science, Langfang 065000, People's Republic of China
}

\section{A R T I C L E I N F O}

\section{Article history:}

Received 13 June 2008

Received in revised form 14 November 2008

Accepted 17 November 2008

Available online 27 December 2008

\section{Keywords:}

China

Polychlorinated biphenyls

Respirable particulate matter

Soil

\begin{abstract}
A B S T R A C T
As one of China's great metropolises, Taiyuan is affected by heavy chemical industry and manufacture of chemical products, and faces pollution from polychlorinated biphenyls (PCBs). Therefore, this study was conducted to determine the PCB concentrations in various environmental media in Taiyuan. We collected 15 soil samples, 34 respirable particulate matter (PM) samples (17 of $\mathrm{PM}_{2.5}$ and 17 of $\mathrm{PM}_{10}$ ) from urban areas of Taiyuan, and measured a total of 144 PCB congeners (including some coeluting PCB congeners). The total PCB concentrations were $51-4.7 \times 10^{3} \mathrm{pg} \mathrm{g}^{-1}$ in soil, $27-1.4 \times 10^{2} \mathrm{pg} \mathrm{m}^{-3}$ in $\mathrm{PM}_{2.5}$ and $16-1.9 \times$ $10^{2} \mathrm{pg} \mathrm{m}^{-3}$ in $\mathrm{PM}_{10}$. Of the PCB homologues, the dominant PCBs detected in the various media were all tri-CBs. Soil was relatively the most polluted media. Furthermore, principal-component analysis revealed that the major PCB source in Taiyuan may be associated with the main commercial PCB through longrange transmission. Toxic equivalency (TEQ) concentrations (based on ten dioxin-like PCBs) ranged from

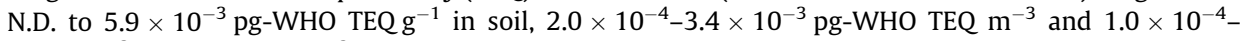
$1.2 \times 10^{-3} \mathrm{pg}$-WHO TEQ $\mathrm{m}^{-3}$ in $\mathrm{PM}_{2.5}$ and $\mathrm{PM}_{10}$, respectively. In previous studies, PCBs were not a severe component of contaminant in Taiyuan; however, this study suggested there is a potential threat of human exposure to PCBs for residents of Taiyuan.
\end{abstract}

(c) 2008 Elsevier Ltd. All rights reserved.

\section{Introduction}

Polychlorinated biphenyls (PCBs) were listed along with 11 other compounds as persistent organic pollutants (POPs) by the Stockholm Convention on 22 May 2001 (The Secretariat of the Stockholm Convention and UNEPs Information Unit for Conventions, 2005). PCBs are stable in the environment, can undergo long-range atmospheric transport, and can bioaccumulate through the food chain (UNECE, 1998; UNEP, 1998). PCBs are a class of 209 congeners that were widely used in a variety of electrical applications such as dielectric fluid in transformers and large capacitors, heat transfer fluids and hydraulic fluids. The carcinogenic and mutagenic properties of PCBs have been extensively studied (Breivik et al., 2002). Most research on environmental contamination from PCBs in China has focused on the eastern part of the country, especially estuarial areas such as those of the Pearl and Yangtze Rivers, Bohai Bay in northern China, and along other major rivers such as the Minjiang, Jiulongjiang, Yangtze, and Songhua Rivers (Xing et al., 2005a). However, the contamination status in terrestrial ecosystems, and especially in urban areas should not be ignored.

\footnotetext{
* Corresponding author. Tel.: +86 10 62919177; fax: +86 1062923563 .

E-mail address: xuxb1006@sina.com (X.-B. Xu).
}

Soil and air are the important components of the terrestrial ecosystem. Soil is generally a primary sink and emission source for contaminants (Duarte-Davidson et al., 1997). Atmospheric particulates are important in contaminant transport over long distances in the terrestrial ecosystem (Hoff et al., 1998). The most significant exposure route of PCB is diet, and almost $95 \%$ of total daily intake (TDI) is taken up by dietary ingestion (Japan Ministry of the Environment, 2003). Direct human exposure via inhalation of outdoor air is not a significant exposure pathway, with respirable particulate matter (PM) the vector by which POPs enter the upper respiratory tract. Fine and ultra fine particles can reach lung alveoli, which is responsible for human exposure (Tasdemir et al., 2004; Kampa and Castanas, 2008). Since PCBs can easily accumulate on particles, contamination of soil and respirable PM have a direct impact on public health and increase the risk of human exposure via inhalation and ingestion (Tasdemir et al., 2004; Kampa and Castanas, 2008). Therefore it is important to monitor and improve understanding of various environmental media that influence breathing concentrations of such contaminants. Since the ratification of the Stockholm Convention (The Secretariat of the Stockholm Convention and UNEPs Information Unit for Conventions, 2005), it has become especially important to control PCBs in China. However, little is known about recent levels of PCBs in various environmental media of Chinese urban areas (Ren et al., 2007; Wang et al., 2008). 
As a metropolis, Taiyuan in Shanxi Province is probably one of the best-known industrial bases in China, with a long history of industrial activity, iron and steel industry, coal-fired power plants, and several coking and chemical plants around the urban area. Rapid economic growth and urbanization in recent years have sharply aggravated its pollution and raised a series of pollution problems. Thus it is necessary to immediately investigate the current contamination status of this key urban area in China.

Because of the paucity of data on PCB levels from various environmental media in Chinese cities (Meijer et al., 2003; Wang et al., 2008), we undertook this study to determine both the concentration and profile of PCBs in soil and respirable PM $\left(\mathrm{PM}_{2.5}\right.$ and $\mathrm{PM}_{10}$ ) samples from Taiyuan urban areas. The main objectives were to establish the urban area's contamination status, explore the interaction of PCBs among these environmental media in the terrestrial ecosystem and identify possible sources of pollution.

\section{Materials and methods}

\subsection{Samples}

Taiyuan city occupies an area of $6956 \mathrm{~km}^{2}$ with $180 \mathrm{~km}^{2}$ classified as urban. The urban area is mainly in the eastern part of Taiyuan and has three million residents. China clay is the main representative soil type in Taiyuan. The climate is dominated by temperate semi-wet monsoon, with a mean annual temperature of $-5.2{ }^{\circ} \mathrm{C}$ during the sampling period.

Sampling sites were set to represent the area of Taiyuan. The sampling locations were chosen by a symmetrical grid $\left(25 \mathrm{~km}^{2}\right.$ per grid), in an attempt to evenly distribute sampling over the region. Surface soil $(0-10 \mathrm{~cm})$ were obtained from 15 sites (site 1-15) in Taiyuan city, Shanxi province (Fig. 1), in January 2006. Surface soil $(0-10 \mathrm{~cm})$ was sampled in triplicate using a hand-held coring device from 15 sites (sites 1-15) in Taiyuan (Fig. 1), in Jan- uary 2006. Each composite soil sample was 16 sub-samples from corresponding grids of sampling sites (Fig. 1). Overlying vegetation was removed prior to collection of the sample. The samples were freeze-dried, thoroughly mixed, sieved to 60-mesh, transferred to pre-cleaned amber glass and maintained at $-18{ }^{\circ} \mathrm{C}$ before analysis. The soil water content was determined gravimetrically after ovendrying individual composite soil samples at $105^{\circ} \mathrm{C}$ for $12 \mathrm{~h}$.

Sampling was done in combination with the PCB soil study, using the same sampling sites. From the corresponding grid for soil samples (sites 1-17, Fig. 1) in Taiyuan, 34 respirable PM $\left(\mathrm{PM}_{2.5}\right.$ and $\mathrm{PM}_{10}$ ) samples were taken in December 2006. To combine with the PCB soil study, the PM sampling was also done in winter. The PM samples were taken with a modified medium-volume TH-150 sampler with dual sampling module, which is in two main parts, filter holder and glass fiber filters, located on the top of the sampler (Wuhan Tianhong Instrument Factory, Wuhan, China). The overall average volume was approximately $24 \mathrm{~m}^{3}$ (average flow rate: $100 \mathrm{~L} \mathrm{~min}^{-1}$ ) and each sample was a $4 \mathrm{~h}$ composite. There were 17 of $\mathrm{PM}_{2.5}$ and 17 of $\mathrm{PM}_{10}$ samples taken. Before the experiment, the fiber filters were annealed for $4 \mathrm{~h}$ at $550^{\circ} \mathrm{C}$ to remove organic material and equilibrated in desiccators, weighed and put into acetone pre-washed aluminous envelopes. After sampling, the filters were removed from the inlet and folded in half and returned to the pre-clean aluminous envelopes and stored them at $-18^{\circ} \mathrm{C}$ before analysis.

\subsection{Chemicals}

The standard solutions of PCB congeners were purchased from AccuStandard Inc. (New Haven, CT, USA). The five PCB calibration mixtures were diluted, which provided 144 congeners, with iso-octane to $20 \mathrm{ng} \mathrm{mL}^{-1}$ for each congener, which provided 144 congeners. Table 1 shows the 144 PCB congeners, which represent the commercial mixtures of PCBs most widely used, such as Aroclor

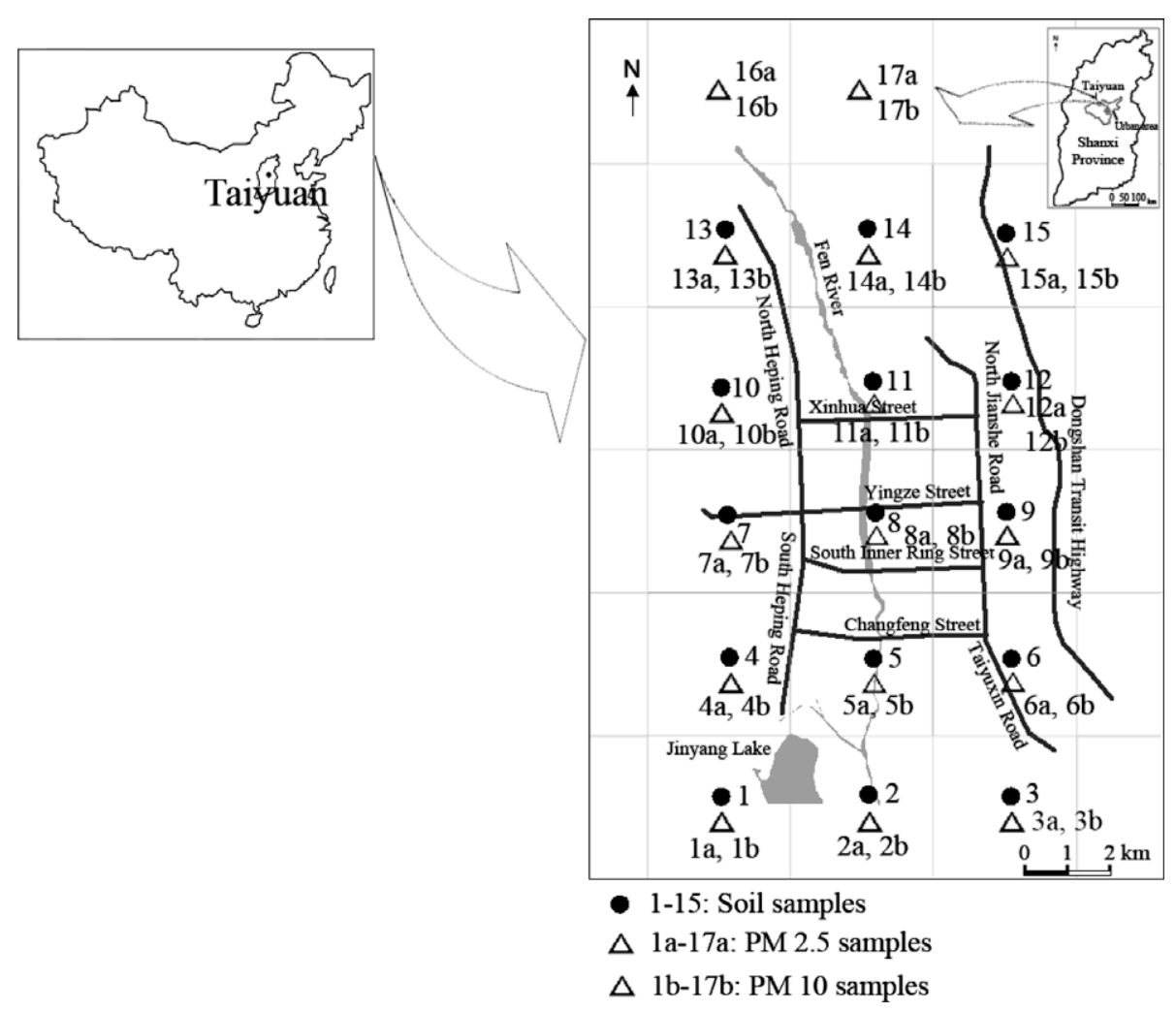

Fig. 1. Sampling sites of various environmental media in urban areas of Taiyuan. 
Table 1

Acquisition data for GC-MS analysis of 144 PCB congeners including two selected ions for each homologue and relevant isotopic ratios.

\begin{tabular}{|c|c|c|c|c|}
\hline $\begin{array}{l}\text { PCB } \\
\text { homologue }\end{array}$ & IUPAC number & $\begin{array}{l}\text { Characteristic fragment } \\
\text { ions MS-1 }\end{array}$ & $\begin{array}{l}\text { Characteristic fragment } \\
\text { ions MS-2 }\end{array}$ & $\begin{array}{l}\text { Isotopic } \\
\text { ratios }\end{array}$ \\
\hline mono-CBs & $1,2,3$ & 188 & 190 & 3.13 \\
\hline di-CBs & $4 / 10,9 / 7,6,8 / 5,14,12 / 13,15$ & 222 & 224 & 1.56 \\
\hline tri-CBs & $19,30,18,17,24 / 27,16 / 32,34,29,26,25,31,28,21,33 / 20,22$ & 256 & 258 & 1.04 \\
\hline tetra-CBs & $\begin{array}{l}54,53,51,45,46,69,52 / 73,49,47 / 75 / 48,44,59 / 42,71 / 41 / 64,40,67,63,74,70,66,56 / 60 \\
81,77\end{array}$ & 290 & 292 & 0.77 \\
\hline penta-CBs & $\begin{array}{l}104,103,100,93,95,91,92,84,90 / 101,99,119,83,97,117 / 87 / 115,85,110,82,124,107 / \\
109,123,118,114,122,105\end{array}$ & 326 & 328 & 1.55 \\
\hline hexa-CBs & $\begin{array}{l}136,154,151,135 / 144,147,149,134,165,146,153,132,141,137,130,164 / 163 / 138,158 \\
129,128,167,156,157\end{array}$ & 360 & 362 & 1.24 \\
\hline hepta-CBs & $179,176,178,175,187,183,185,174,177,171,173,172,180 / 193,191,170 / 190,189$ & 394 & 396 & 1.05 \\
\hline octa-CBs & 202, 201, 197, 200, 199, 203/196, 195, 194, 205 & 428 & 430 & 0.89 \\
\hline nona-CBs & $208,207,206$ & 462 & 464 & 0.77 \\
\hline dec-CB & 209 & 496 & 498 & 0.69 \\
\hline
\end{tabular}

1242, 1254, 1260, 1268 (World Health Organization (WHO), 1993). Decachlorobiphenyl (CB-209), used as a surrogate, was purchased from Supelco (Bellfonte, PA, USA). The working standards were prepared by diluting the above-mentioned standard solution with iso-octane. Standard reference material GBW08307 (reference soil for PCBs congeners analysis, including CB-28, 52, 77, 81, 101, 118, $114,105,138,153,167,156,157$ and 180) was obtained from the National Research Center for Certified Reference Materials of China. The laboratory glassware was washed with detergents and dichromate acid cleaning solution, rinsed with tap water and distilled water, and then rinsed with methanol (analytical grade, Beijing Chemical Factory, China), dichloromethane (analytical grade, Beijing Chemical Factory, China) and acetone (analytical grade, Beijing Chemical Factory, China), respectively. The pre-clean procedure of each solvent was repeated six times using fresh solvent for the laboratory glassware prior to use. The other solvents used were pesticide grade (J.T. Baker, Phillipsburg, NJ, USA).

\subsection{Extraction and cleanup}

Five grams of each soil sample or each PM sample was ground with anhydrous sodium sulfate into a free-flowing powder. Each sample was extracted with $30 \mathrm{~mL}$ of hexane/dichloromethane $(1: 1, \mathrm{vol} / \mathrm{vol})$ by ultrasonication for $4 \mathrm{~min}$ and then centrifuged at $3000 \mathrm{~g}$. This process was repeated three times and the extracts were combined. Before extraction, $100 \mu \mathrm{L}$ of $\mathrm{CB}-209\left(100 \mathrm{ng} \mathrm{mL}^{-1}\right)$ was added in each sample as a surrogate standard and balanced at desiccator for $2 \mathrm{~h}$. The concentrated extracts were evaporated to $1 \mathrm{~mL}$ in a Kuderna-Danish concentrator under a gentle $\mathrm{N}_{2}$ stream for cleanup.

The concentrated extracts were cleaned by using a chromatography column $(20 \mathrm{~cm} \times 15-\mathrm{mm}$ internal diameter $)$ containing the following: $2 \mathrm{~g}$ of silver nitrate silica (10\% concentrated silver nitrate, wt/wt), $1 \mathrm{~g}$ of activated silica gel, $3 \mathrm{~g}$ of basic silica gel, $1 \mathrm{~g}$ of activated silica gel, $4 \mathrm{~g}$ of acid silica gel (44\% concentrated sulfuric acid, wt/wt), $4 \mathrm{~g}$ of acid silica gel ( $22 \%$ concentrated sulfuric acid, wt/wt), $1 \mathrm{~g}$ of activated silica gel and $2 \mathrm{~g}$ of anhydrous sodium sulfate. The PCB fraction was eluted with $100 \mathrm{~mL}$ of hexane. The elution was then evaporated with a rotary evaporator and then reduced to $50 \mu \mathrm{L}$ under a gentle $\mathrm{N}_{2}$ stream for analysis.

\subsection{Analysis}

PCBs were measured with an Agilent 6890 series gas chromatograph (GC) coupled with an Agilent 5973 mass spectrometer (MS) using electron impact ionization source in SIM mode. In electron impact ionization mode, the MS source temperature was $230^{\circ} \mathrm{C}$, and the electron energy was $70 \mathrm{eV}$. The GC was equipped with a split- splitless injector held constant at $270^{\circ} \mathrm{C}$. Gas chromatographic separation was performed on a DB-5MS capillary column (30 $\mathrm{m} \times 0.25$ $\mathrm{mm}$ internal diameter; $0.25-\mu \mathrm{m}$ film thickness). The GC column was maintained at $75^{\circ} \mathrm{C}$ for $2 \mathrm{~min}$ and then ramped at $15^{\circ} \mathrm{C} \mathrm{min}-1$ to $150{ }^{\circ} \mathrm{C}$, further ramped at $2.5^{\circ} \mathrm{C} \mathrm{min}{ }^{-1}$ to $280^{\circ} \mathrm{C}$, and held at this temperature for $15 \mathrm{~min}$. The total run time was $64 \mathrm{~min}$.

\subsection{Quality control}

All analytical procedures were monitored using strict quality assurance and control measures. Field blanks consisted of preclean soil and pre-clean glass fiber filters which were taken to the sampling site. A total of six field blanks were extracted and analyzed in the same way as samples. The target compounds were not detected when the field blank samples were analyzed. A solvent blank, a procedural blank and a standard mixture were run per 10 samples to check for contamination, peak identification and quantification. The target compounds were not detected in the solvent blanks and the procedural blanks. Duplicate samples in the laboratory were analyzed along with regular samples to determine repeatability and reproducibility, for additional quality-control assessment to ensure valid results. Instrument stability and relative response factor variance were determined by analyzing the calibration standard solutions in each sample batch.

PCBs were quantified using an external standard method. Three quality-control criteria were used to ensure correct identification of the target compounds: (1) GC retention times matched those of the standard compounds within $0.05 \mathrm{~min}$; (2) the signal-tonoise ratio was greater than $3: 1$; and (3) each compound had two monitored ions. Isotopic ratios between quantitative and confirmation ions were within $15 \%$ of theoretical values. In the present study, some coeluting PCB congeners exist, and they were shown in Tables 1 and 2 (such as 4/10, 9/7, 8/5, 12/13 etc.). Coeluting $\mathrm{PCB}$ congeners were reported as individual results in Table 2. For example, PCB 9 and PCB 7 coeluted, so if the value of that coelution was 10 , then the records for PCB 9 and PCB 7 will be shown with a single record containing the parameter name "РCB 9/7" and the quantitative value "10". The limits of detection for PCBs were defined by a signal-to-noise ratio greater than three times the average baseline variation and were within $0.01-0.05 \mathrm{ng} \mathrm{g}^{-1}$ (dry weight). The matrix spike recoveries of the 144 PCB congeners (including some coeluting PCB congeners) were within $70-110 \%$, with $10 \%$ standard deviation. The recovery of CB-209 surrogate in all samples was within the range of $70-105 \%$.

The standard reference material sample including 14 PCB congeners (GBW 08307, National Research Center for Certified Reference Materials, China) was analyzed to validate the analytical method used. The results were satisfactory, with a $z$ score $\leqslant 1$ for 
Table 2

Measured PCB concentrations in soil, $\mathrm{PM}_{2.5}$ and $\mathrm{PM}_{10}$.

\begin{tabular}{|c|c|c|c|c|c|c|c|c|c|c|c|c|}
\hline \multirow{2}{*}{ 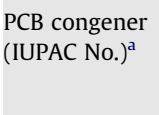 } & \multicolumn{4}{|c|}{ Soil $\left(\mathrm{pg} \mathrm{g}^{-1}, n=15\right)$} & \multicolumn{4}{|c|}{$\mathrm{PM}_{2.5}\left(\mathrm{pg} \mathrm{m}^{-3}, n=17\right)$} & \multicolumn{4}{|c|}{$\mathrm{PM}_{10}\left(\mathrm{pg} \mathrm{m}^{-3}, n=17\right)$} \\
\hline & Minimum & Maximum & Median & $\begin{array}{l}\text { Standard } \\
\text { deviation }\end{array}$ & Minimum & Maximum & Median & $\begin{array}{l}\text { Standard } \\
\text { deviation }\end{array}$ & Minimum & Maximum & Median & $\begin{array}{l}\text { Standard } \\
\text { deviation }\end{array}$ \\
\hline 1 & N.D. ${ }^{b}$ & 63 & N.D. & 16 & N.D. & 1.7 & N.D. & 0.6 & N.D. & 1.9 & 1.1 & 0.8 \\
\hline 2 & N.D. & 81 & N.D. & 26 & N.D. & 0.9 & N.D. & 0.2 & N.D. & 0.6 & N.D. & 0.2 \\
\hline 3 & N.D. & 75 & N.D. & 24 & N.D. & 6.4 & N.D. & 2.0 & N.D. & 4.5 & N.D. & 1.1 \\
\hline $4 / 10$ & N.D. & N.D. & N.D. & N.D. & N.D. & N.D. & N.D. & N.D. & N.D. & N.D. & N.D. & N.D. \\
\hline $9 / 7$ & N.D. & 72 & N.D. & 19 & N.D. & 1.0 & N.D. & 0.2 & N.D. & N.D. & N.D. & N.D. \\
\hline 6 & N.D. & 80 & N.D. & 28 & N.D. & 2.0 & N.D. & 0.8 & N.D. & 2.2 & N.D. & 0.8 \\
\hline 8 & N.D. & $1.5 \times 10^{2}$ & N.D. & 53 & N.D. & 6.7 & N.D. & 2.1 & N.D. & 12 & N.D. & 4.3 \\
\hline 5 & N.D. & $1.1 \times 10^{2}$ & N.D. & 37 & N.D. & 5.4 & 2.9 & 1.7 & N.D. & 7.4 & 3.1 & 2.3 \\
\hline 14 & N.D. & 10 & N.D. & 2.7 & N.D. & 0.4 & N.D. & 0.1 & N.D. & N.D. & N.D. & N.D. \\
\hline $12 / 13$ & N.D. & N.D. & N.D. & N.D. & N.D. & N.D. & N.D. & N.D. & N.D. & N.D. & N.D. & N.D. \\
\hline 15 & N.D. & 46 & N.D. & 13 & N.D. & 95 & N.D. & 23 & N.D. & 90 & N.D. & 24 \\
\hline 19 & N.D. & 12 & N.D. & 5.5 & N.D. & 1.0 & N.D. & 0.4 & N.D. & 2.0 & 0.5 & 0.6 \\
\hline 30 & N.D. & $3.4 \times 10^{2}$ & 66 & 86 & 1.3 & 5.6 & 3.4 & 1.1 & 1.6 & 5.6 & 3.7 & 1.2 \\
\hline 18 & N.D. & $4.5 \times 10^{2}$ & 58 & $1.2 \times 10^{2}$ & N.D. & 7.4 & 1.9 & 2.0 & 1.0 & 6.9 & 3.3 & 1.9 \\
\hline 17 & N.D. & 18 & N.D. & 5.0 & N.D. & 0.5 & N.D. & 0.2 & N.D. & 1.3 & N.D. & 0.4 \\
\hline $24 / 27$ & N.D. & $3.2 \times 10^{2}$ & N.D. & 83 & N.D. & 3.1 & N.D. & 1.2 & N.D. & 7.2 & N.D. & 2.1 \\
\hline $16 / 32$ & N.D. & 70 & N.D. & 27 & N.D. & 3.4 & N.D. & 1.1 & N.D. & 4.0 & 1.6 & 1.7 \\
\hline 34 & N.D. & 17 & N.D. & 5.9 & N.D. & 1.2 & N.D. & 0.4 & N.D. & 0.6 & N.D. & 0.3 \\
\hline 29 & N.D. & 11 & N.D. & 3.8 & N.D. & 0.7 & N.D. & 0.2 & N.D. & 0.8 & N.D. & 0.3 \\
\hline $26 / 25$ & N.D. & 9.5 & N.D. & 3.1 & N.D. & 0.8 & 0.3 & 0.3 & N.D. & 1.0 & N.D. & 0.4 \\
\hline 31 & N.D. & $1.2 \times 10^{2}$ & 23 & 38 & N.D. & 6.2 & 1.7 & 1.5 & N.D. & 4.2 & 1.9 & 1.1 \\
\hline 28 & N.D. & $2.6 \times 10^{2}$ & N.D. & 68 & N.D. & 2.4 & N.D. & 0.7 & N.D. & 2.4 & N.D. & 0.6 \\
\hline 21 & N.D. & 19 & N.D. & 5.6 & N.D. & 12 & N.D. & 2.8 & N.D. & 12 & N.D. & 2.9 \\
\hline $33 / 20$ & N.D. & 34 & N.D. & 11 & N.D. & N.D. & N.D. & N.D. & N.D. & 2.7 & N.D. & 0.9 \\
\hline 22 & N.D. & 30 & N.D. & 7.7 & N.D. & 0.8 & N.D. & 0.3 & N.D. & 0.7 & N.D. & 0.2 \\
\hline 54 & N.D. & 17 & N.D. & 4.4 & N.D. & 0.6 & N.D. & 0.2 & N.D. & N.D. & N.D. & N.D. \\
\hline 53 & N.D. & 22 & N.D. & 5.7 & N.D. & N.D. & N.D. & N.D. & N.D. & 0.9 & N.D. & 0.3 \\
\hline 51 & N.D. & 81 & N.D. & 21 & N.D. & 5.1 & 1.0 & 1.7 & N.D. & 3.9 & 1.3 & 1.2 \\
\hline 45 & N.D. & 24 & N.D. & 10 & N.D. & 3.9 & N.D. & 1.0 & N.D. & 0.9 & N.D. & 0.2 \\
\hline 46 & N.D. & 24 & N.D. & 8.5 & N.D. & 0.8 & N.D. & 0.3 & N.D. & 2.1 & N.D. & 0.6 \\
\hline 69 & N.D. & 16 & N.D. & 7.0 & N.D. & 0.9 & N.D. & 0.2 & N.D. & 1.0 & N.D. & 0.3 \\
\hline $52 / 73$ & N.D. & $1.1 \times 10^{2}$ & N.D. & 30 & N.D. & N.D. & N.D. & N.D. & N.D. & 2.1 & N.D. & 0.7 \\
\hline 49 & N.D. & $2.2 \times 10^{2}$ & N.D. & 60 & N.D. & 1.1 & 0.5 & 0.4 & N.D. & 2.5 & N.D. & 0.7 \\
\hline 47 & N.D. & $1.2 \times 10^{2}$ & N.D. & 34 & N.D. & N.D. & N.D. & N.D. & N.D. & 1.0 & N.D. & 0.2 \\
\hline $75 / 48$ & N.D. & $1.1 \times 10^{2}$ & N.D. & 35 & N.D. & 18 & N.D. & 6.0 & N.D. & 15 & N.D. & 3.6 \\
\hline 44 & N.D. & 29 & N.D. & 10 & N.D. & 0.7 & N.D. & 0.2 & N.D. & 1.2 & N.D. & 0.4 \\
\hline 59 & N.D. & 29 & N.D. & 10 & N.D. & 28 & N.D. & 6.7 & N.D. & 3.1 & N.D. & 0.9 \\
\hline 42 & N.D. & 54 & N.D. & 16 & N.D. & N.D. & N.D. & N.D. & N.D. & 1.2 & N.D. & 0.4 \\
\hline $71 / 41$ & N.D. & 25 & N.D. & 9.1 & N.D. & 0.9 & N.D. & 0.3 & N.D. & 1.6 & N.D. & 0.5 \\
\hline 64 & N.D. & 14 & N.D. & 4.6 & N.D. & 0.6 & N.D. & 0.3 & N.D. & 1.0 & N.D. & 0.3 \\
\hline 40 & N.D. & N.D. & N.D. & N.D. & N.D. & 11 & N.D. & 2.8 & N.D. & 1.0 & N.D. & 0.2 \\
\hline 67 & N.D. & 16 & N.D. & 5.3 & N.D. & 12 & N.D. & 3.0 & N.D. & 0.5 & N.D. & 0.2 \\
\hline 63 & N.D. & 13 & N.D. & 3.3 & N.D. & 0.7 & N.D. & 0.3 & N.D. & 0.8 & N.D. & 0.3 \\
\hline 74 & N.D. & 22 & N.D. & 7.3 & N.D. & 1.7 & 0.4 & 0.4 & N.D. & 1.5 & N.D. & 0.4 \\
\hline 70 & N.D. & $3.9 \times 10^{2}$ & N.D. & $1.0 \times 10^{2}$ & N.D. & 1.8 & 0.4 & 0.5 & N.D. & 3.1 & N.D. & 0.8 \\
\hline 66 & N.D. & 44 & N.D. & 12 & N.D. & 2.9 & N.D. & 0.7 & N.D. & 0.8 & N.D. & 0.3 \\
\hline $56 / 60$ & N.D. & 29 & N.D. & 9.9 & N.D. & 2.8 & N.D. & 0.7 & N.D. & 1.5 & 0.4 & 0.4 \\
\hline 81 & N.D. & 11 & N.D. & 2.9 & N.D. & 10 & 0.5 & 2.5 & N.D. & 2.1 & 0.7 & 0.6 \\
\hline 77 & N.D. & 20 & N.D. & 7.8 & N.D. & 3.9 & 0.8 & 1.0 & N.D. & 2.2 & 0.6 & 0.5 \\
\hline 104 & N.D. & N.D. & N.D. & N.D. & N.D. & N.D. & N.D. & N.D. & N.D. & 0.6 & N.D. & 0.1 \\
\hline 103 & N.D. & N.D. & N.D. & N.D. & N.D. & N.D. & N.D. & N.D. & N.D. & 0.8 & N.D. & 0.2 \\
\hline 100 & N.D. & N.D. & N.D. & N.D. & N.D. & 0.7 & N.D. & 0.2 & N.D. & 6.7 & N.D. & 1.6 \\
\hline 93 & N.D. & N.D. & N.D. & N.D. & N.D. & N.D. & N.D. & N.D. & N.D. & 0.9 & N.D. & 0.2 \\
\hline 95 & N.D. & N.D. & N.D. & N.D. & N.D. & 1.0 & N.D. & 0.4 & N.D. & 0.9 & N.D. & 0.3 \\
\hline 91 & N.D. & 15 & N.D. & 4.0 & N.D. & 0.7 & N.D. & 0.2 & N.D. & 1.6 & N.D. & 0.4 \\
\hline $92 / 84$ & N.D. & 24 & N.D. & 6.2 & N.D. & N.D. & N.D. & N.D. & N.D. & 1.6 & N.D. & 0.4 \\
\hline 90 & N.D. & $1.3 \times 102$ & N.D. & 35 & N.D. & 2.1 & N.D. & 0.6 & N.D. & 1.0 & N.D. & 0.3 \\
\hline 101 & N.D. & $2.3 \times 10^{2}$ & N.D. & 64 & N.D. & 1.4 & N.D. & 0.4 & N.D. & 1.3 & N.D. & 0.4 \\
\hline 99 & N.D. & 22 & N.D. & 5.6 & N.D. & 2.5 & N.D. & 0.7 & N.D. & 0.9 & N.D. & 0.3 \\
\hline 119 & N.D. & 12 & N.D. & 3.0 & N.D. & 0.8 & N.D. & 0.3 & N.D. & 1.0 & N.D. & 0.3 \\
\hline 83 & N.D. & N.D. & N.D. & N.D. & N.D. & 1.3 & N.D. & 0.5 & N.D. & 1.8 & N.D. & 0.6 \\
\hline 97 & N.D. & 29 & N.D. & 9.8 & N.D. & 1.0 & N.D. & 0.3 & N.D. & 1.2 & N.D. & 0.3 \\
\hline 117 & N.D. & 17 & N.D. & 4.3 & N.D. & 3.6 & N.D. & 0.9 & N.D. & 0.9 & N.D. & 0.3 \\
\hline 87 & N.D. & N.D. & N.D. & N.D. & N.D. & 0.8 & N.D. & 0.3 & N.D. & 0.8 & N.D. & 0.3 \\
\hline 115 & N.D. & 81 & N.D. & 21 & N.D. & 0.8 & N.D. & 0.3 & N.D. & 0.8 & N.D. & 0.3 \\
\hline 85 & N.D. & 27 & N.D. & 9.3 & N.D. & 1.1 & N.D. & 0.3 & N.D. & 1.3 & N.D. & 0.4 \\
\hline $110 / 82$ & N.D. & $6.2 \times 10^{2}$ & 20 & $1.6 \times 10^{2}$ & N.D. & 17 & N.D. & 4.1 & N.D. & 6.5 & 0.9 & 1.7 \\
\hline 124 & N.D. & 45 & N.D. & 14 & N.D. & 1.1 & N.D. & 0.4 & N.D. & 2.5 & N.D. & 0.7 \\
\hline 107 & N.D. & 16 & N.D. & 4.2 & N.D. & 1.2 & N.D. & 0.4 & N.D. & 1.0 & N.D. & 0.4 \\
\hline $109 / 123$ & N.D. & 15 & N.D. & 4.0 & N.D. & 2.3 & N.D. & 0.9 & N.D. & 1.7 & N.D. & 0.8 \\
\hline 118 & N.D. & N.D. & N.D. & N.D. & N.D. & 1.3 & N.D. & 0.5 & N.D. & 10 & 0.6 & 2.5 \\
\hline 114 & N.D. & 53 & N.D. & 14 & N.D. & 1.9 & 0.6 & 0.6 & N.D. & 2.1 & 0.7 & 0.6 \\
\hline 122 & N.D. & N.D. & N.D. & N.D. & N.D. & 1.9 & N.D. & 0.6 & N.D. & 2.1 & 0.7 & 0.6 \\
\hline
\end{tabular}


Table 2 (continued)

\begin{tabular}{|c|c|c|c|c|c|c|c|c|c|c|c|c|}
\hline \multirow{2}{*}{$\begin{array}{l}\text { PCB congener } \\
\left(_{\text {IUPAC No. })^{\mathrm{a}}}\right.\end{array}$} & \multicolumn{4}{|c|}{ Soil $\left(\mathrm{pg} \mathrm{g}^{-1}, n=15\right)$} & \multicolumn{4}{|c|}{$\mathrm{PM}_{2.5}\left(\mathrm{pg} \mathrm{m}^{-3}, n=17\right)$} & \multicolumn{4}{|c|}{$\mathrm{PM}_{10}\left(\mathrm{pg} \mathrm{m}^{-3}, n=17\right)$} \\
\hline & Minimum & Maximum & Median & $\begin{array}{l}\text { Standard } \\
\text { deviation }\end{array}$ & Minimum & Maximum & Median & $\begin{array}{l}\text { Standard } \\
\text { deviation }\end{array}$ & Minimum & Maximum & Median & $\begin{array}{l}\text { Standard } \\
\text { deviation }\end{array}$ \\
\hline 105 & N.D. & 18 & N.D. & 4.7 & N.D. & 2.1 & 0.7 & 0.6 & N.D. & 1.8 & 0.6 & 0.6 \\
\hline 136 & N.D. & 19 & N.D. & 4.8 & N.D. & N.D. & N.D. & N.D. & N.D. & N.D. & N.D. & N.D. \\
\hline 154 & N.D. & 24 & N.D. & 6.2 & N.D. & N.D. & N.D. & N.D. & N.D. & N.D. & N.D. & N.D. \\
\hline 151 & N.D. & N.D. & N.D. & N.D. & N.D. & 0.6 & N.D. & 0.2 & N.D. & N.D. & N.D. & N.D. \\
\hline 135 & N.D. & 20 & N.D. & 5.0 & N.D. & 0.9 & N.D. & 0.2 & N.D. & 0.9 & N.D. & 0.2 \\
\hline 144 & N.D. & 21 & N.D. & 6.3 & N.D. & 0.9 & N.D. & 0.2 & N.D. & 0.6 & N.D. & 0.2 \\
\hline 147 & N.D. & 20 & N.D. & 5.0 & N.D. & 0.8 & N.D. & 0.2 & N.D. & 0.6 & N.D. & 0.2 \\
\hline 149 & N.D. & $2.0 \times 10^{2}$ & 14 & 57 & N.D. & 0.8 & N.D. & 0.2 & N.D. & 0.8 & N.D. & 0.2 \\
\hline 134 & N.D. & 22 & N.D. & 5.7 & N.D. & 0.9 & N.D. & 0.2 & N.D. & 0.9 & N.D. & 0.2 \\
\hline 165 & N.D. & 16 & N.D. & 4.2 & N.D. & 0.8 & N.D. & 0.2 & N.D. & 3.2 & N.D. & 0.8 \\
\hline 146 & N.D. & 14 & N.D. & 4.5 & N.D. & 0.7 & N.D. & 0.2 & N.D. & 1.3 & N.D. & 0.3 \\
\hline 153 & N.D. & 11 & N.D. & 4.5 & N.D. & 1.7 & N.D. & 0.4 & N.D. & 1.0 & N.D. & 0.2 \\
\hline $132 / 141$ & N.D. & $3.2 \times 10^{2}$ & N.D. & 93 & N.D. & 2.0 & N.D. & 0.6 & N.D. & 1.9 & N.D. & 0.6 \\
\hline 137 & N.D. & 26 & N.D. & 8.5 & N.D. & N.D. & N.D. & N.D. & N.D. & N.D. & N.D. & N.D. \\
\hline 130 & N.D. & 32 & N.D. & 11 & N.D. & 0.9 & N.D. & 0.3 & N.D. & 1.3 & N.D. & 0.5 \\
\hline 164 & N.D. & 34 & N.D. & 10 & N.D. & 0.8 & N.D. & 0.3 & N.D. & 1.1 & N.D. & 0.3 \\
\hline $163 / 138$ & N.D. & $3.3 \times 10^{2}$ & N.D. & 87 & N.D. & 1.0 & N.D. & 0.3 & N.D. & 1.1 & N.D. & 0.3 \\
\hline 158 & N.D. & 52 & N.D. & 14 & N.D. & 0.4 & N.D. & 0.1 & N.D. & 0.6 & N.D. & 0.2 \\
\hline 129 & N.D. & 44 & N.D. & 14 & N.D. & 0.8 & N.D. & 0.3 & N.D. & 0.9 & N.D. & 0.3 \\
\hline 128 & N.D. & 17 & N.D. & 5.6 & N.D. & 1.1 & N.D. & 0.4 & N.D. & 1.1 & N.D. & 0.4 \\
\hline 167 & N.D. & 14 & N.D. & 5.5 & N.D. & 3.5 & N.D. & 0.9 & N.D. & 1.1 & N.D. & 0.3 \\
\hline 156 & N.D. & 40 & N.D. & 11 & N.D. & 1.6 & 0.2 & 0.5 & N.D. & 1.4 & 0.6 & 0.4 \\
\hline 157 & N.D. & 27 & N.D. & 10 & N.D. & 1.1 & 0.5 & 0.4 & N.D. & 0.9 & 0.4 & 0.3 \\
\hline 179 & N.D. & 14 & N.D. & 4.3 & N.D. & N.D. & N.D. & N.D. & N.D. & 0.4 & N.D. & 0.1 \\
\hline 176 & N.D. & N.D. & N.D. & N.D. & N.D. & 0.7 & N.D. & 0.2 & N.D. & 0.6 & N.D. & 0.2 \\
\hline 178 & N.D. & 19 & N.D. & 4.9 & N.D. & N.D. & N.D. & N.D. & N.D. & 0.9 & N.D. & 0.3 \\
\hline 175 & N.D. & 14 & N.D. & 3.6 & N.D. & N.D. & N.D. & N.D. & N.D. & 0.8 & N.D. & 0.3 \\
\hline 187 & N.D. & 57 & N.D. & 15 & N.D. & N.D. & N.D. & N.D. & N.D. & N.D. & N.D. & N.D. \\
\hline 183 & N.D. & 16 & N.D. & 5.2 & N.D. & 0.8 & N.D. & 0.2 & N.D. & N.D. & N.D. & N.D. \\
\hline 185 & N.D. & 18 & N.D. & 7.0 & N.D. & 1.4 & N.D. & 0.3 & N.D. & 0.7 & N.D. & 0.2 \\
\hline 174 & N.D. & 20 & N.D. & 6.4 & N.D. & 0.6 & N.D. & 0.2 & N.D. & 0.6 & N.D. & 0.2 \\
\hline 177 & N.D. & 14 & N.D. & 3.5 & N.D. & 0.6 & N.D. & 0.2 & N.D. & 0.6 & N.D. & 0.1 \\
\hline 171 & N.D. & 20 & N.D. & 6.7 & N.D. & 1.1 & N.D. & 0.3 & N.D. & N.D. & N.D. & N.D. \\
\hline 173 & N.D. & 18 & N.D. & 6.1 & N.D. & N.D. & N.D. & N.D. & N.D. & 0.6 & N.D. & 0.2 \\
\hline 172 & N.D. & 22 & N.D. & 7.1 & N.D. & N.D. & N.D. & N.D. & N.D. & 0.7 & N.D. & 0.2 \\
\hline $180 / 193$ & N.D. & 96 & 11 & 29 & N.D. & 1.5 & N.D. & 0.4 & N.D. & 0.8 & N.D. & 0.3 \\
\hline 191 & N.D. & 12 & N.D. & 3.0 & N.D. & 0.5 & N.D. & 0.1 & N.D. & 0.9 & N.D. & 0.2 \\
\hline $170 / 190$ & N.D. & $1.1 \times 10^{2}$ & N.D. & 29 & N.D. & 2.3 & N.D. & 0.6 & N.D. & 0.8 & N.D. & 0.3 \\
\hline 189 & N.D. & 27 & N.D. & 9.3 & N.D. & 0.8 & N.D. & 0.3 & N.D. & 1.5 & N.D. & 0.4 \\
\hline 202 & N.D. & 14 & N.D. & 3.7 & N.D. & 0.7 & N.D. & 0.2 & N.D. & 1.0 & N.D. & 0.4 \\
\hline 201 & N.D. & 23 & N.D. & 8.6 & N.D. & N.D. & N.D. & N.D. & N.D. & 1.2 & N.D. & 0.3 \\
\hline 197 & N.D. & 27 & N.D. & 9.5 & N.D. & 0.9 & N.D. & 0.3 & N.D. & 0.8 & N.D. & 0.3 \\
\hline 200 & N.D. & 13 & N.D. & 3.3 & N.D. & 1.0 & N.D. & 0.2 & N.D. & 0.7 & N.D. & 0.2 \\
\hline 199 & N.D. & 33 & N.D. & 11 & N.D. & 1.0 & N.D. & 0.3 & N.D. & 0.7 & N.D. & 0.2 \\
\hline 203/196 & N.D. & 22 & N.D. & 8.1 & N.D. & 1.6 & N.D. & 0.4 & N.D. & 1.0 & N.D. & 0.4 \\
\hline 195 & N.D. & 28 & N.D. & 10 & N.D. & 0.9 & N.D. & 0.3 & N.D. & 0.8 & N.D. & 0.3 \\
\hline 194 & N.D. & 56 & 11.8 & 16 & N.D. & 1.3 & N.D. & 0.4 & N.D. & 1.1 & N.D. & 0.4 \\
\hline 205 & N.D. & 55 & N.D. & 17 & N.D. & 1.0 & N.D. & 0.4 & N.D. & 1.1 & N.D. & 0.4 \\
\hline 208 & N.D. & 32 & N.D. & 10 & N.D. & 1.1 & N.D. & 0.4 & N.D. & 0.9 & N.D. & 0.3 \\
\hline 207 & N.D. & 20 & N.D. & 6.8 & N.D. & N.D. & N.D. & N.D. & N.D. & 1.4 & N.D. & 0.3 \\
\hline 206 & N.D. & 41 & N.D. & 16 & N.D. & 1.7 & N.D. & 0.5 & N.D. & 1.4 & N.D. & 0.5 \\
\hline
\end{tabular}

a The number used to designate each congener by International Union of Pure and Applied Chemistry (IUPAC).

${ }^{b}$ N.D. was calculated assuming non-detected measurements were equal to LOD.

all congeners (range of $0.02-0.64$ for the PCBs; $p<0.05$ ). Recovery of CB-209 surrogate was $85 \%$ with the GBW 08307 sample.

\subsection{Data analysis}

Statistical analysis was done using SPSS 13.0 for Windows (SPSS, Chicago, IL, USA). We used principal-component analysis (PCA), a multivariate statistical technique, to derive new components (PCs) as a linear combination of the original variables, where PCs attempt to preserve the statistical relationships of the original data. For this study, we investigated interspecies variations in PCB congeners using PCA. Initial variables considered were the concentrations of PCB homologues. We normalized values of PCBs to a percentage of the sum of all congeners. Varimax rotation in the PCA was used to facilitate interpretation.

\section{Results and discussion}

\subsection{PCBs in soil}

PCBs (144 congeners, including some coeluting PCB congeners) were identified in 15 urban soil samples (Table 2). The detection rates of PCBs in urban soils reached $100 \%$, which indicates wide occurrence in Taiyuan (Fig. 2A). The total PCB concentrations (defined as the sum of 144 congeners) were $51-4.7 \times 10^{3} \mathrm{pg} \mathrm{g}^{-1}$ (median: $6.4 \times 10^{2} \mathrm{pg} \mathrm{g}^{-1}$; dry weight; Table 3 ). These PCB levels were compared with soil concentrations of PCBs reported elsewhere (Ma et al., 2007; Ren et al., 2007; Fu et al., 2008; Wang et al., 2008). The median concentration in the present study was much lower than those obtained in global background soil (5.4 $\mathrm{ng} \mathrm{g}^{-1}$, dry weight) (Meijer et al., 2003) and was similar to 

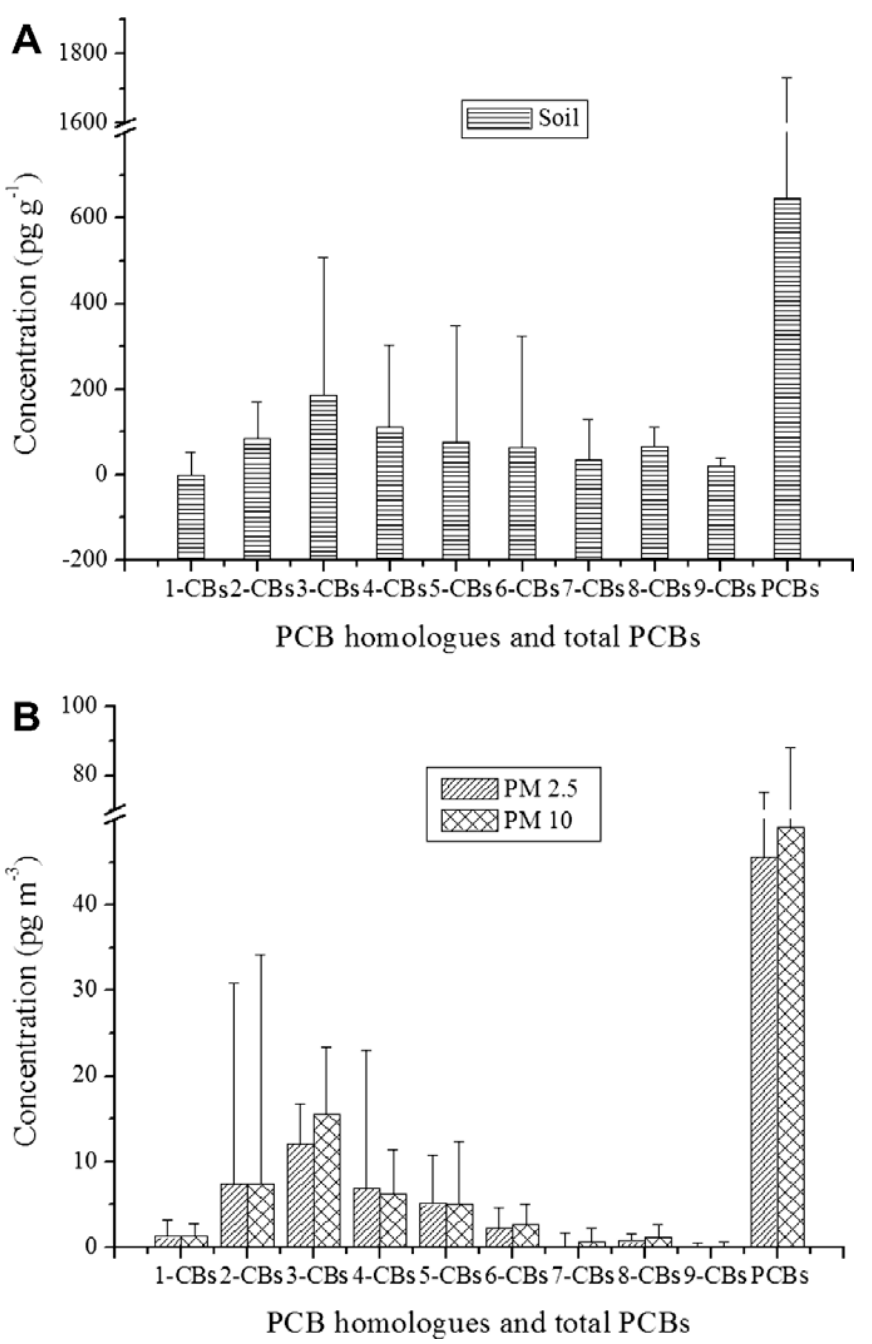

Fig. 2. Distribution of $\mathrm{PCB}$ homologues and $\Sigma \mathrm{PCB}$ from various environmental media in Taiyuan (A: Soil; B: $\mathrm{PM}_{2.5}$ and $\mathrm{PM}_{10}$ ).

those obtained in surface soil of China (median: 515 pg g $^{-1}$, dry weight) (Ren et al., 2007). In comparison with other areas of China, the levels were higher than those in suburban soil of Beijing $\left(0.18 \mathrm{ng} \mathrm{g}^{-1}\right.$ ) (Chu et al., 1995); and lower than those in soil of Shenyang (6.4-15.2 $\mathrm{ng} \mathrm{g}^{-1}$ ), a major industrial city in north China (Jing et al., 1992), the surface soil of Dalian (2.8 $\mathrm{ng} \mathrm{g}^{-1}$, dry weight) (Wang et al., 2008), and soil of the chemical industrial zone in Shanghai (0.5-586.85 $\mathrm{ng} \mathrm{g}^{-1}$ ) (Ma et al., 2007). Compared to other countries, the levels were lower than those in surface soil of mainland Europe (97 $\mathrm{ng} \mathrm{g}^{-1}$ ) (Meijer et al., 2003) and Spain (4.4-4.7 $\mathrm{ng} \mathrm{g}^{-1}$ ) (Nadal et al., 2007), and were similar to those in the soil (0.6-5.1 $\left.\mathrm{ng} \mathrm{g}^{-1}\right)$ of the chemical/petrochemical industrial zone of Tarragona County (Nadal et al., 2008). According to the allowable level of $20 \mu \mathrm{g} \mathrm{kg}^{-1}$ for the total concentration of six PCB congeners approved by the Dutch List (VROM, 2000), the soil in our study generally had relatively low PCB contamination.

Of the PCB homologues, the dominant PCBs detected from soil in this study were tri-CBs (median: $28.8 \%$ ), followed by tetra-CBs and di-CBs (medians: $17.2 \%$ and $13.1 \%$, respectively; Fig. 2A). Higher-chlorinated congeners from soil were higher than those obtained from respirable PM in Taiyuan. This profile is reasonable since higher-chlorinated congeners are firmly bound to particles due to their low volatility (Chu et al., 1995).

\section{2. $P C B$ in respirable $P M$}

There were 144 PCBs (including some coeluting PCB congeners) identified in 34 respirable PM samples (17 of $\mathrm{PM}_{2.5}$ and 17 of $\mathrm{PM}_{10}$, respectively; Table 2 ). The total PCB concentrations were 27-1.4 $\times$ $10^{2} \mathrm{pg} \mathrm{m}^{-3}$ (median: $46 \mathrm{pg} \mathrm{m}^{-3}$ ) in $\mathrm{PM}_{2.5}$, and $16-1.9 \times 10^{2} \mathrm{pg} \mathrm{m}^{-3}$ (median: $49 \mathrm{pg} \mathrm{m}^{-3}$ ) in $\mathrm{PM}_{10}$ (Table 3 ). The median concentrations in $\mathrm{PM}_{2.5}$ and $\mathrm{PM}_{10}$ in Taiyuan were similar. There are few previous studies on $\mathrm{PM}_{2.5}$ and $\mathrm{PM}_{10}$ (Helm and Bidleman, 2005; Lammel et al., 2007; Mandalakis and Stephanou, 2007). Furthermore, atmospheric pollution directly influences respirable PM. Therefore, we compared the atmospheric concentrations of PCBs reported elsewhere (Cheng et al., 2007; Lammel et al., 2007; Mandalakis and Stephanou, 2007; Siddik Cindoruk and Tasdemir, 2007). In comparison with other areas of China, the median concentrations in the present study were lower than those from urban areas in the Yellow and East China Seas region (360 $\mathrm{pg} \mathrm{m}^{-3}$ and 297-537 $\mathrm{pg} \mathrm{m}^{-3}$, respectively) (Lammel et al., 2007), chemical industrial zone in Shanghai (2017.22 $\mathrm{pg} \mathrm{m}^{-3}$ ) (Cheng et al., 2007). Compared to air from other countries, on the other hand, the levels were similar to those in the atmosphere of Melpitz $\left(110 \pm 80 \mathrm{pg} \mathrm{m}^{-3}\right)$, a rural site in eastern Germany (Mandalakis and Stephanou, 2007), and were lower than those in the air from an urban area of Bursa, Turkey $\left(491.8 \pm 189.4 \mathrm{pg} \mathrm{m}^{-3}\right.$ ) (Siddik Cindoruk and Tasdemir, 2007).

Of PCB homologues, the dominant PCBs in $\mathrm{PM}_{2.5}$ were similar to those in $\mathrm{PM}_{10}$. The dominant PCBs detected from $\mathrm{PM}_{2.5}$ and $\mathrm{PM}_{10}$ in this study were also tri-CBs, accounting for medians of $26.5 \%$ and $31.5 \%$, respectively, followed by di-CBs and tetra-CBs (Fig. 2B). Higher-chlorinated congeners were detected at relatively low concentrations. This profile was expected since the lower volatility of higher-chlorinated congeners mean they become more firmly bound in heavily polluted areas (Chu et al., 1995). In other areas in China, tri-CBs were also the main $\mathrm{PCB}$ homologues in air samples (36.03-42.73\%) of the Pearl River Delta (close to the City of Guangzhou, China) (Li et al., 2007). In urban air samples of Europe, the major PCB homologues were tetra-PCBs and tri-PCBs (Howel, 2007).

\subsection{PCB spatial character}

There were some correlations between soil and respirable PM $\left(\mathrm{PM}_{2.5}\right.$ and $\left.\mathrm{PM}_{10}\right)$. Concentration of $\Sigma \mathrm{PCB}$ showed that soil was relatively the most polluted media, followed by $\mathrm{PM}_{2.5}$ and $\mathrm{PM}_{10}$ (Table 3). According to each media, the differences in concentration might be neglected when accounting for errors in measurement, extraction and cleanup. These results indicated that in Taiyuan the PCB distributions in soil, $\mathrm{PM}_{2.5}$ and $\mathrm{PM}_{10}$ were quite uniform. This may be due to the limited production of industrial PCBs and their relatively short application time in China.

In the present study, it was clear that the more chlorinated biphenyl congeners were higher in soil than in $\mathrm{PM}_{2.5}$ and $\mathrm{PM}_{10}$, possibly due to their low volatility. Of PCB homologues, the triPCBs were dominant in all media in this study. Thus, soil was not only an important sink but also a key source that influenced respirable PM.

Toxic equivalency (TEQ) concentrations in soil and respirable $\mathrm{PM}\left(\mathrm{PM}_{2.5}\right.$ and $\left.\mathrm{PM}_{10}\right)$ were calculated using World Health Organization Toxic Equivalent Factors (WHO-TEFs) (Van den Berg, 2006; Bhavsar et al., 2008). In the present study, TEQ concentrations based on 10 dioxin-like PCBs (PCB-77, PCB-81, PCB-105, PCB-114, PCB-118, PCB-123, PCB-156, PCB-157, PCB-167 and PCB-189). In the present study, PCB-81 was the predominant dioxin-like PCB, followed by $\mathrm{PCB}-77$. The concentrations of TEQs ranged from N.D. to $5.9 \times 10^{-3} \mathrm{pg}^{-W H O} \mathrm{TEQ} \mathrm{g}^{-1}$ in soil, $2.0 \times 10^{-4}-3.4 \times$ $10^{-3} \mathrm{pg}$-WHO TEQ $\mathrm{m}^{-3}$ in $\mathrm{PM}_{2.5}$ and $1.0 \times 10^{-4}-1.2 \times 10^{-3} \mathrm{pg}$ WHO TEQ $\mathrm{m}^{-3}$ in $\mathrm{PM}_{10}$, respectively (Table 3 ). Moreover, the most 
Table 3

Total concentrations of PCB homologues and TEQs in each sample.

\begin{tabular}{|c|c|c|c|c|c|c|c|c|c|c|c|}
\hline Sample & mono-CBs & di-CBs & tri-CBs & tetra-CBs & penta-CBs & hexa-CBs & hepta-CBs & octa-CBs & nona-CBs & $\Sigma$ PCBs & TEQ \\
\hline \multicolumn{12}{|c|}{ Soil (the data of PCB concentrations are in $\mathrm{pg} \mathrm{g}^{-1}$; the data of TEQs are in $p g-W H O T E Q g^{-1}$ ) } \\
\hline 1 & N.D. ${ }^{\mathrm{a}}$ & 78 & $2.4 \times 10^{2}$ & 39 & $1.3 \times 10^{2}$ & 86 & 65 & 94 & 57 & $7.8 \times 10^{2}$ & N.D. \\
\hline 2 & 63 & N.D. & $1.1 \times 10^{2}$ & 90 & 25 & 20 & 12 & $1.2 \times 10^{2}$ & 54 & $4.9 \times 10^{2}$ & $1.5 \times 10^{-3}$ \\
\hline 3 & N.D. & 85 & 36 & 49 & 38 & 46 & N.D. & 17 & 21 & $2.9 \times 10^{2}$ & $2.0 \times 10-3$ \\
\hline 4 & N.D. & 88 & $1.7 \times 10^{2}$ & 68 & $4.7 \times 10^{2}$ & $4.5 \times 10^{2}$ & 36 & N.D. & N.D. & $1.3 \times 10^{3}$ & $1.8 \times 10^{-3}$ \\
\hline 5 & N.D. & N.D. & 22 & N.D. & N.D. & N.D. & N.D. & 11 & 19 & 51 & N.D. \\
\hline 6 & $2.0 \times 10^{2}$ & $1.7 \times 10^{2}$ & $2.7 \times 10^{2}$ & 60 & $1.2 \times 10^{2}$ & 13 & 11 & N.D. & 31 & $8.7 \times 10^{2}$ & N.D. \\
\hline 7 & N.D. & $2.9 \times 10^{2}$ & $4.6 \times 10^{2}$ & $1.8 \times 10^{2}$ & 26 & $3.3 \times 10^{2}$ & $1.4 \times 10^{2}$ & 48 & N.D. & $1.5 \times 10^{2}$ & $3.0 \times 10^{-3}$ \\
\hline 8 & 78 & N.D. & $1.3 \times 10^{3}$ & $7.6 \times 10^{2}$ & $1.1 \times 10^{3}$ & $9.8 \times 10^{2}$ & $3.6 \times 10^{2}$ & 88 & 24 & $4.7 \times 10^{3}$ & $3.1 \times 10^{-3}$ \\
\hline 9 & N.D. & $1.0 \times 10^{2}$ & 84 & $1.1 \times 10^{2}$ & 95 & $2.7 \times 10^{2}$ & $1.6 \times 10^{2}$ & $1.2 \times 10^{2}$ & 36 & $9.8 \times 10^{2}$ & $6.0 \times 10^{-4}$ \\
\hline 10 & 23 & N.D. & $4.0 \times 10^{2}$ & $2.0 \times 10^{2}$ & 76 & $1.3 \times 10^{2}$ & 12 & 67 & N.D. & $9.2 \times 10^{2}$ & $5.9 \times 10^{-3}$ \\
\hline 11 & N.D. & 98 & $1.4 \times 10^{2}$ & $1.6 \times 10^{2}$ & $1.7 \times 10^{2}$ & 64 & 73 & $1.0 \times 10^{2}$ & N.D. & $8.1 \times 10^{2}$ & $1.3 \times 10^{-3}$ \\
\hline 12 & N.D. & N.D. & $2.3 \times 10^{2}$ & $1.1 \times 10^{2}$ & 20 & 10 & N.D. & N.D. & N.D. & $3.7 \times 10^{2}$ & $2.1 \times 10^{-3}$ \\
\hline 13 & N.D. & $1.4 \times 10^{2}$ & $1.4 \times 10^{2}$ & $2.6 \times 10^{2}$ & N.D. & 24 & 24 & 69 & N.D. & $6.5 \times 10^{2}$ & N.D. \\
\hline 14 & N.D. & $1.5 \times 10^{2}$ & $5.1 \times 10^{2}$ & $4.0 \times 10^{2}$ & $2.1 \times 10^{2}$ & $2.0 \times 10^{2}$ & $1.0 \times 10^{2}$ & 43 & 34 & $1.6 \times 10^{3}$ & $1.1 \times 10^{-3}$ \\
\hline 15 & N.D. & N.D. & $1.9 \times 10^{2}$ & $2.3 \times 10^{2}$ & 28 & 54 & 56 & 74 & 21 & $6.5 \times 10^{2}$ & $3.1 \times 10^{-3}$ \\
\hline \multicolumn{12}{|c|}{$P M_{2.5}$ (the data of PCB concentrations are in $\mathrm{pg} \mathrm{m}^{-3}$; the data of TEQs are in $\mathrm{pg}$-WHO TEQ $\mathrm{m}^{-3}$ ) } \\
\hline 1 & 1.3 & 4.2 & 6.7 & 4.5 & 22 & 4.7 & 6.9 & 1.6 & N.D. & 52 & $5.0 \times 10^{-4}$ \\
\hline 2 & 1.5 & 1.5 & 13 & 21 & 11 & 8.9 & 3.7 & 0.8 & 1.1 & 63 & $3.4 \times 10^{-3}$ \\
\hline 3 & 0.4 & 3.0 & 12 & 69 & 8.7 & 7.9 & 0.7 & N.D. & N.D. & $1.0 \times 10^{2}$ & $9.0 \times 10^{-4}$ \\
\hline 4 & N.D. & 13 & 8.1 & 22 & 11 & 2.7 & N.D. & N.D. & 1.7 & 58 & $5.0 \times 10^{-4}$ \\
\hline 5 & N.D. & 14 & 5.4 & 3.0 & N.D. & 2.3 & 0.8 & 1.7 & 1.4 & 28 & $2.0 \times 10^{-4}$ \\
\hline 6 & 7.3 & 4.0 & 12 & 20 & 5.2 & 3.5 & N.D. & 2.9 & N.D. & 55 & $1.0 \times 10^{-4}$ \\
\hline 7 & 3.0 & 12 & 4.2 & 6.9 & N.D. & 1.4 & N.D. & N.D. & N.D. & 27 & $3.0 \times 10^{-4}$ \\
\hline 8 & 0.6 & 5.1 & 9.4 & 7.6 & 6.5 & 2.0 & 1.1 & N.D. & N.D. & 32 & $2.0 \times 10^{-4}$ \\
\hline 9 & 2.7 & $1.0 \times 10^{2}$ & 23 & 4.1 & 7.3 & 1.2 & N.D. & 1.4 & N.D. & $1.4 \times 10^{2}$ & $2.0 \times 10^{-4}$ \\
\hline 10 & 4.5 & 14 & 9.7 & 6.6 & 7.7 & 1.8 & 0.6 & 0.6 & N.D. & 46 & $2.0 \times 10^{-4}$ \\
\hline 11 & 1.1 & 8.7 & 13 & 25 & 1.2 & 0.6 & N.D. & N.D. & N.D. & 50 & $4.0 \times 10^{-4}$ \\
\hline 12 & 2.4 & 2.0 & 11 & 7.5 & 1.6 & 2.0 & N.D. & 1.5 & N.D. & 28 & $3.0 \times 10^{-4}$ \\
\hline 13 & 1.7 & 7.4 & 20 & 5.7 & 4.1 & 2.0 & 0.8 & 1.0 & N.D. & 42 & $2.0 \times 10^{-4}$ \\
\hline 14 & N.D. & 14 & 11 & 6.0 & 13 & 5.4 & 0.6 & 0.6 & N.D. & 50 & $3.0 \times 10^{-4}$ \\
\hline 15 & N.D. & 6.0 & 15 & 7.6 & 4.9 & 3.1 & N.D. & 1.7 & 1.0 & 39 & $2.0 \times 10^{-4}$ \\
\hline 16 & N.D. & 11 & 13 & 5.3 & 4.0 & 2.8 & N.D. & 2.3 & N.D. & 38 & $2.0 \times 10^{-4}$ \\
\hline 17 & 1.3 & 5.2 & 13 & 5.5 & 3.1 & 0.5 & N.D. & 0.6 & N.D. & 29 & $3.0 \times 10^{-4}$ \\
\hline \multicolumn{12}{|c|}{$P M_{10}$ (the data of PCB concentrations are in $\mathrm{pg} \mathrm{m}^{-3}$; the data of TEQs are in $\mathrm{pg}$-WHO TEQ $\mathrm{m}^{-3}$ ) } \\
\hline 1 & 1.9 & 9.3 & 11 & 5.4 & 16 & 9.3 & 6.5 & 0.6 & N.D. & 60 & $7.0 \times 10^{-4}$ \\
\hline 2 & N.D. & 4.3 & 13 & 5.2 & 13 & 2.0 & 2.6 & 2.2 & 2.3 & 45 & $2.0 \times 10^{-4}$ \\
\hline 3 & N.D. & 4.4 & 8.2 & 7.3 & 24 & 6.8 & 1.6 & 1.5 & N.D. & 53 & $8.0 \times 10^{-4}$ \\
\hline 4 & 5.8 & 6.6 & 12 & 5.0 & 4.1 & 0.7 & 1.2 & 0.9 & 1.4 & 38 & $3.0 \times 10^{-4}$ \\
\hline 5 & N.D. & 3.3 & 5.8 & 4.0 & 2.9 & 0.5 & N.D. & N.D. & N.D. & 16 & $1.0 \times 10^{-4}$ \\
\hline 6 & 0.6 & 3.7 & 6.3 & 9.3 & 3.8 & 6.3 & 1.1 & 3.4 & N.D. & 35 & $5.0 \times 10^{-4}$ \\
\hline 7 & 1.3 & 54 & 16 & 10 & 4.1 & 2.3 & N.D. & 1.1 & N.D. & 88 & $5.0 \times 10^{-4}$ \\
\hline 8 & N.D. & 18 & 18 & 5.7 & 6.2 & 2.0 & 1.3 & 0.6 & 0.9 & 53 & $2.0 \times 10^{-4}$ \\
\hline 9 & 1.7 & N.D. & 22 & 11 & 13 & 1.6 & N.D. & N.D. & N.D. & 49 & $4.0 \times 10^{-4}$ \\
\hline 10 & 2.3 & 7.2 & 8.6 & 6.2 & 5.4 & 2.7 & 0.5 & 2.7 & N.D. & 36 & $6.0 \times 10^{-4}$ \\
\hline 11 & 1.1 & 5.8 & 14 & 5.0 & 3.7 & 3.6 & 0.7 & N.D. & N.D. & 34 & $4.0 \times 10^{-4}$ \\
\hline 12 & N.D. & 13 & 18 & 24 & 4.7 & 3.9 & N.D. & 4.5 & N.D. & 68 & $5.0 \times 10^{-4}$ \\
\hline 13 & 1.8 & $1.1 \times 10^{2}$ & 40 & 13 & 26 & 2.1 & N.D. & 2.7 & N.D. & $1.9 \times 10^{2}$ & $1.2 \times 10^{-3}$ \\
\hline 14 & 1.8 & 26 & 19 & 15 & 5.1 & 1.9 & N.D. & N.D. & N.D. & 68 & $2.0 \times 10^{-4}$ \\
\hline 15 & 3.2 & 7.4 & 17 & 6.6 & 7.0 & 2.9 & N.D. & 1.3 & 0.7 & 46 & $2.0 \times 10^{-4}$ \\
\hline 16 & 1.1 & 10 & 18 & 5.5 & 1.7 & 4.1 & 0.9 & 3.3 & N.D. & 44 & $2.0 \times 10^{-4}$ \\
\hline 17 & 1.7 & 18 & 17 & 4.6 & 4.7 & 2.8 & 0.8 & 1.2 & 1.4 & 53 & $3.0 \times 10^{-4}$ \\
\hline
\end{tabular}

${ }^{a}$ N.D. was calculated assuming non-detected measurements were equal to LOD.

significant uptake route of dioxin-like PCBs is the diet (Japan Ministry of the Environment, 2003), with almost 95\% of TDI via food contamination. Inhalation intake doses have been reported to contribute 2.61\% to the TDI (Japan Ministry of the Environment, 2003). Due to lack of data about dioxin-like PCB diet exposure, we cannot calculate total daily dioxin-like $\mathrm{PCB}$ intake doses of Taiyuan citizens.

\subsection{Source analysis}

To evaluate similarities or differences between the PCB homologue patterns of various media samples, we performed PCA on data normalized to percentage of $\Sigma$ PCBs. PCs were determined by an eigenvalue $>1$. By processing, two extracted PCs explained $70.3 \%$ of the data variance. A loading plot and score plot were obtained after varimax rotation. Component 1 accounted for $54.6 \%$ of the total variance and was characterized by higher-chlorinated homologues, whereas component 2 accounted for $15.7 \%$ and was characterized by lower-chlorinated homologues. The score plot (Fig. 3) indicated that the compositions of PCBs in the various media samples were similar, possibly originating from the same source. The PCBs found in Taiyuan could therefore be classified into one main group that was relatively highly contaminated by tri-CBs. There was also a clear clustering of samples, showing that tri-CBs were the dominant contaminant in the soil, $\mathrm{PM}_{2.5}$ and $\mathrm{PM}_{10}$

In China, approximately $10000 \mathrm{t}$ of PCBs were produced in 1965-1974, with World Health Organization (WHO), 1993. Polychlorinated biphenyls and terphenyls (2nd ed.), environmental health criteria 140. Geneva. Nine thousand tonnes of trichlorobiphenyl (the main PCB) and $1000 \mathrm{t}$ of pentachlorobiphenyl (the \#2 PCB) (Xing et al., 2005b); he former contained 42\% chlorine, similar to Aroclor 1242 , and the latter $53 \%$ chlorine, similar to 


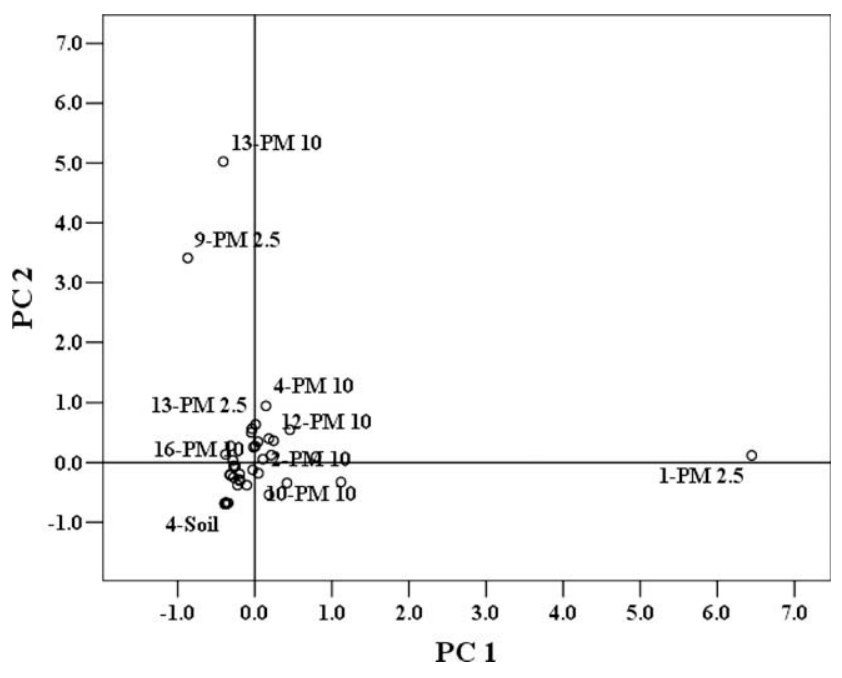

Fig. 3. Two-dimensional principal-component score plot obtained from the data correlation matrix of 15 soil samples, 17 samples of $\mathrm{PM}_{2.5}$ and 17 samples of $\mathrm{PM}_{10}$ samples in Taiyuan.

Aroclor 1254 (Jiang et al., 1997). The major homologues produced and used in China was tri-CBs, the major homologue profile of global PCB products was also composed of tri-CBs, and the composition of tri-CBs ( $40.4 \%$ of total) was higher in Chinese products than in globally (25.2\% of total) (Breivik et al., 2002). Therefore, tri-CBs should be the dominant PCBs in soil, $\mathrm{PM}_{2.5}$ and $\mathrm{PM}_{10}$ of Taiyuan. All these samples as well as the major commercial PCB were characterized by a higher proportion of tri-CBs in the $\mathrm{PCB}$ profiles, which indicates that the sources of PCBs for these sampling locations may be associated with the main commercial PCB through long-range transmission.

\section{Conclusions}

The present work investigated the composition, distribution and characterization of PCBs in soil and respirable PM. The results indicate that the sources of PCBs for these sampling locations may be associated with the main commercial PCB through long-range transmission. Although the PCB contamination in various environmental media in Taiyuan was low, there was a potential threat to human health. All the findings indicate that attention should be paid to PCB contamination of the urban environment to maintain human health and environmental safety in China.

\section{Acknowledgements}

This study was supported by the National Natural Scientific Foundation of China (No. 20707031). We thank the research staff in the Inst. Geochem. Geophys. Exploration, and especially Prof. Cheng and Prof. Liu for sample collection.

\section{References}

Bhavsar, S.P., Reiner, E.J., Hayton, A., Fletcher, R., MacPherson, K., 2008. Converting toxic equivalents (TEQ) of dioxins and dioxin-like compounds in fish from one toxic equivalency factor (TEF) scheme to another. Environ. Int. 34, 915-921.

Breivik, K., Sweetman, A., Pacyna, J.M., Jones, K.C., 2002. Towards a global historical emission inventory for selected PCB congeners - a mass balance approach 1 . Global production and consumption. Sci. Total Environ. 290, 181-198.

Cheng, J.P., Wu, Q., Xie, H.Y., Gu, J.M., Zhao, W.C., Ma, J., Wang, W.H., 2007. Polychlorinated biphenyls (PCBs) in $\mathrm{PM}_{10}$ surrounding a chemical industrial zone in Shanghai, China. Bull. Environ. Contam. Toxicol. 79, 448-453.
Chu, S.G., Yang, C., Xu, X.B., Liu, X.X., 1995. Polychlorinated biphenyl congener residues in sediment and soil from pollution area. China Environ. Sci. 15, 199203.

Duarte-Davidson, R., Sewart, A., Alcock, R.E., Cousins, I.T., Jones, K.C., 1997. Exploring the balance between sources, deposition, and the environmental burden of PCDD/Fs in the UK terrestrial environment: an aid to identifying uncertainties and research needs. Environ. Sci. Technol. 31, 1-11.

Fu, S., Yang, Z.Z., Li, K., Xu, X.B., 2008. Polychlorinated biphenyl residues in sandstorm depositions in Beijing, China. Chemosphere 73, 962-966.

Helm, P.A., Bidleman, T.F., 2005. Gas-particle partitioning of polychlorinated naphthalenes and non- and mono-ortho-substituted polychlorinated biphenyls in arctic air. Sci. Total Environ. 342, 161-173.

Hoff, R.M., Brice, K.A., Halsall, C.J., 1998. Non-linearity in the slope of clausiusclapeyron plots for SVOCs. Environ. Sci. Technol. 32, 1793-1798.

Howel, D., 2007. Multivariate data analysis of pollutant profiles: PCB levels across Europe. Chemosphere 67, 1300-1307.

Japan Ministry of the Environment, 2003. Information Brochure Dioxins. Government of Japan, Japan. Available from: http://www.env.go.jp/en/topic/ dioxins.html.

Jiang, K., Li, L., Chen, Y., Jin, J., 1997. Determination of PCDD/Fs and dioxin-like PCBs in Chinese commercial PCBs and emissions from a testing PCB incinerator Chemosphere 34, 941-950.

Jing, Z.Y., Li, Y.H., Feng, X.B., Wang, F.Q., Zhang, Y.X., 1992. The study of polychlorinated biphenyls for their loss, contamination and protection strategy in Shanyang City. Congkan Environ. Sci. 13, 1-28 (in Chinese).

Kampa, M., Castanas, E., 2008. Human health effects of air pollution. Environ. Pollut $151,362-367$.

Lammel, G., Ghim, Y.S., Grados, A., Gao, H., Huhnerfuss, H., Lohmann, R., 2007. Levels of persistent organic pollutants in air in China and over the Yellow Sea. Atmos. Environ. 41, 452-464.

Li, C.L., Hao, Y.M., Mai, B.X., Sheng, G.Y., Fu, J.M., 2007. Spatial distribution of atmospheric PCBs in winter Pearl River Delta. Acta Sci. Circums. 27, 655-659.

Ma, J., Cheng, J., Xie, H., Hu, X., Li, W., Zhang, J., Yuan, T., Wang, W., 2007. Seasona and spatial character of PCBs in a chemical industrial zone of Shanghai. China Environ. Geochem. Health 29, 503-511.

Mandalakis, M., Stephanou, E.G., 2007. Atmospheric concentration characteristics and gas-particle partitioning of PCBs in a rural area of eastern Germany. Environ. Pollut. 147, 211-221.

Meijer, S.N., Ockenden, W.A., Sweetman, A., Breivik, K., Grimalt, J.O., Jones, K.C., 2003. Global distribution and budget of PCBs and HCB in background surface soils: implications for sources and environmental processes. Environ. Sci. Technol. 37, 667-672.

Nadal, M., Mari, M., Schuhmacher, M., Domingo, J.L., 2008. Multi-compartmental environmental surveillance of a petrochemical area: levels of micropollutants. Environ. Int.. doi:10.1016/j.envint.2008.1006.1001.

Nadal, M., Schuhmacher, M., Domingo, J.L., 2007. Levels of metals, PCBs, PCNs and PAHs in soils of a highly industrialized chemical/petrochemical area: temporal trend. Chemosphere 66, 267-276.

Ren, N.Q., Que, M.X., Li, Y.F., Liu, Y., Wang, X.N., Xu, D.D., Sverko, E., Ma, J.M., 2007. Polychlorinated biphenyls in Chinese surface soils. Environ. Sci. Technol. 41, 3871-3876.

The Secretariat of the Stockholm Convention and UNEPs Information Unit for Conventions, 2005. Ridding the World of POPs: A Guide to the Stockholm Convention on Persistent Organic Pollutants. UNEP, Geneva, Switzerland. Available from: http://www.pops.int/documents/guidance/beg_guide.pdf.

Siddik Cindoruk, S., Tasdemir, Y., 2007. Characterization of gas/particle concentrations and partitioning of polychlorinated biphenyls (PCBs) measured in an urban site of Turkey. Environ. Pollut. 148, 325-333.

Tasdemir, Y., Odabasi, M., Vardar, N., Sofuoglu, A., Murphy, T.J., Holsen, T.M., 2004 Dry deposition fluxes and velocities of polychlorinated biphenyls (PCBs) associated with particles. Atmos. Environ. 38, 2447-2456.

UNECE, 1998. Protocol on Persistent Organic Pollutants under the 1979 Convention on Long-Range Transboundary Air Pollution (ECE/EB.Air/60). United Nations Economic Commission for Europe, Geneva.

UNEP, 1998. Preparation of an Internationally Legally Binding Instrument for Implementing International Action on Certain Persistent Organic Pollutants; UNEP/POPs/Inc.1/6. United Nations Environment Programme, Nairobi, Kenya.

Van den Berg, M., 2006. The 2005 WHO re-evaluation of toxic equivalency factors for dioxin like compounds - implications for risk assessment and limitations of the concept. Toxicol. Lett. 164, S55-S56.

VROM, 2000. Circular on Target Values and Intervention Values for Soil Remediation. DBO/07494913, VROM (The Housing, Spatial Planning and Environment).

Wang, D.G., Yang, M., Jia, H.L., Zhou, L., Li, Y.F., 2008. Levels, distributions and profiles of polychlorinated biphenyls in surface soils of Dalian, China. Chemosphere 73, 38-42.

World Health Organization (WHO), 1993. Polychlorinated Biphenyls and Terphenyls (2nd ed.), Environmental Health Criteria 140. Geneva.

Xing, Y., Lu, Y., Dawson, R.W., Shi, Y., Zhang, H., Wang, T., Liu, W., Ren, H., 2005a. A spatial temporal assessment of pollution from PCBs in China. Chemosphere 60, 731-739.

Xing, Y., Lu, Y., Dawson, R.W., Shi, Y., Zhang, H., Wang, T., Liu, W., Ren, H., 2005b. A spatial temporal assessment of pollution from PCBs in China. Chemosphere 60, 731-739. 